Journal of Applied Pharmaceutical Science Vol. 5 (10), pp. 107-112, October, 2015

Available online at http://www.japsonline.com

DOI: $10.7324 / \mathrm{JAPS} .2015 .501018$

ISSN 2231-3354 (cc) BY-NC-SA

\title{
Exploring novel drug targets in fatty acid pathway of Plasmodium falciparum
}

\author{
Meenakshi Pradhan ${ }^{1}$, I Arnold Emerson ${ }^{1}$, R Kiruba Thangam², J Febin Prabhu Dass ${ }^{1}$ \\ ${ }^{1}$ Bioinformatics Division, School of Bio Sciences and Technology, VIT University, Vellore 632014, Tamilnadu, India. \\ ${ }^{2}$ Enterprise and Cloud Computing, School of Information Technology \& Engineering, VIT University, Vellore 632014, Tamilnadu, In dia.
}

\author{
ARTICLE INFO \\ Article history: \\ Received on: $21 / 07 / 2015$ \\ Revised on: 20/08/2015 \\ Accepted on: 13/09/2015 \\ Available online: 28/10/2015 \\ Key words : \\ Stoichiometric matrix, \\ Elementary Flux Modes, \\ Drug Target, Malaria. Fatty \\ Acid synthesis pathway.
}

\begin{abstract}
The malarial parasite Plasmodium falciparum infects humans and proliferates rapidly inside the host before its detection. The proliferation step requires a large amount of lipids for membrane synthesis. Thus fatty acid biosynthesis occurring in the apicoplast plays an important role in causing cerebral malaria. In this study, we explored and analyzed these pathways using stoichiometric matrix, elementary flux modes and robustness analysis. Based on the above analysis, the robustness of this pathway diminished as the result of virtual enzyme knock out indicating four key enzymes, 3-oxoacyl-ACP synthase, 3-oxoacyl-ACP synthase, 3-oxoacyl-ACP synthase and Glycerol-3-phosphate o-acyl transferase. Among the four, the first three are existing drug targets. Subsequently, we also found that a combinatorial double knock out of these enzymes predicts further reduction in overall pathway enzyme activity. Thus, we propose multi drug targeting as a better way to treat brain malaria.
\end{abstract}

\section{INTRODUCTION}

Malaria is a vector-borne infectious disease caused by the Plasmodium species. Out of the four types of the Plasmodium parasites that can affect humans, Plasmodium falciparum and Plasmodium vivax are the most dangerous (Das et al., 2009) The other species that can also affect humans are Plasmodium ovale and Plasmodium malariae. These malaria parasites have a complicated life cycle, requiring both a human and a mosquito host, and differentiating multiple times during the transmission process (Aly et al., 2009). The growing parasite requires large amount of lipids for the increase in the parasite surface area and the internal membranes. Also, the parasite is incapable of synthesizing these fatty acids de novo and is restricted to obtain them from the host. This makes the lipid metabolism pathway as a preferred drug target (Ben et al., 2010). However, certain enzymes associated with the type II fatty acid synthesis have

\footnotetext{
* Corresponding Author

J. Febin Prabhu Dass, Associate Professor, Bioinformatics Division, School of Bio Sciences and Technology, VIT University, Vellore 632014, Tamilnadu, India. E-mail: mail2febin@gmail.com
}

been identified in the apicoplast of the parasite. An apicoplast is a non-photosynthetic plastid. It is mostly of red alga origin. The apicoplast has four membranes, and a small cellular genome which codes for the enzymes present in it. This genome is related to algal plastid genome. An important feature of the apicoplast is that it interacts with the environment to import and export many molecules (Lim and McFadden, 2010). The organelle is mostly associated with the biosynthesis of fatty acid, isoprenoid precursors and heme. The parasite is absolutely dependent on this organelle. Most importantly, the cyanobacterial heritage of apicoplast implies that most of its enzymes are different from the mammalian host's equivalents and thus these enzymes can be treated as potential drug targets (Ralph et al., 2004). The apicoplast apparently imports trioses that are either converted into fatty acids or isoprenoid precursors. The apicoplast imports phosphoenol pyruvate (PEP) by phosphoenol pyruvate/ phosphate translocator (PPT). This PEP then becomes the source of carbon and energy for the apicoplast, and also serves as the starting material for the fatty acid biosynthesis. The PEP taken up by the apicoplast is converted to acetyl-CoA through the action of the various enzymes as shown. Acetyl-CoA and pyruvate are the main carbon substrate for fatty acid synthesis in this case. 
The enzymatic activity of the pyruvate dehydreogenase complex involves three co-factors-lipoic acid, thiamine pyrophosphate (TPP) and coenzyme A (CoA) (Ralph et al., 2004). The conversion of acetyl CoA to malonyl-CoA is the first committed step of the pathway and is also considered as the rate limiting step. Both acetyl-CoA and malonyl-CoA are substrates for type II fatty acid synthase (FAS). The various FAS enzymes characterized in this pathway are- Malonyl-CoA transacylase (FabD), B-ketoacyl-ACP synthase III (FabH), Enoyl-ACP reductase (FabI), ß-ketoacyl-ACP reductase (FabG), ßhydroxyacyl-ACP dehydratase (FabZ) and B-ketoacyl-ACP synthase I/II (FabB/F). FabH functions in initiation, FabB and FabF function in elongation. These are isoforms of the same enzyme and differ in their biochemical properties (Waller et al., 2003). The three major products of the pathway are palmitate, palmitoyl-CoA and diacyl-glycerol-3-phosphate. These compounds are released from here and enter various other metabolic pathways that help in the development of the parasite. For example, palmitoyl-CoA enters the GPI anchorage biosynthesis and plays a major role in the formation of the GPI protein. Both palmitate and diacyl-glycerol-3-phosphate enter phosphatidyl serine metabolism. These are important pathways for the parasite development and the inhibition of the fatty acid biosynthesis will result in the overall inhibition of the parasite (Qidwai and Khan, 2012).

The stable functioning of the living being is dependent on the metabolic pathways. This creates a connection between the various metabolites and their functions. The metabolic pathways are basically of two types- anabolic pathways and catabolic pathways. Study of the metabolic networks can give us a fair idea about the actual process going on inside any given cell or tissue. It gives us information about the various metabolites involved along with their status as the substrate or the product. This study has become relatively easier since the genomic era (Papin et al., 2003). Along with that, the growth in the information technology has given rise to the development of various tools which can analyze and interpret any pathway in ways that would be hard to achieve and very expensive in wet lab experiments. Metabolic pathway analysis is used to define the structure and the functional capabilities of the organism. It uses flux balance analysis (Kauffman et al., 2003), stoichiometric analysis and the reversibility status of the reactions involved to analyze the pathway. This analysis can be used to determine the path of an infection, find drug targets, study the effectiveness of the drug targets, increase or decrease the production of certain metabolite to combat the disease at molecular level.

\section{MATERIALS AND METHODS}

\section{Metabolic pathway data}

Apicoplast is a plastid-like organelle found in Apicomplexan parasites. The de-novo fatty acid biosynthesis pathway present in apicoplast shows great potential as target for small molecule inhibitors in standalone and combination therapy.
The apicomplexan cellular mechanisms are likely to be similar in most parasites but their metabolic machinery depends on specific environmental conditions they adapt to. We have obtained the fatty acid biosynthesis taking place in the apicoplast of the Plasmodium falciparum from Malaria Parasite Metabolic Pathways (http://mpmp.huji.ac.il/). This pathway includes 42 metabolites and 30 reactions.

\section{Metatool}

\section{For Studying Metabolic Networks}

The stoichiometric and thermodynamic feasibility of the pathway has to be tested to reconstruct a metabolic network from biochemical data. This is achieved by characterizing admissible region of flux distribution in steady state in which intermediate or internal metabolites are balanced. We define enzymes subsets to be group of enzymes that operate together in fixed flux proportions in all steady states of the system. METATOOL is a novel computer program in which algorithms for calculating convex basis, stoichiometric matrix and enzyme subsets are implemented (Pfeiffer et al., 1999).

\section{Yet Another Network Analyzer (YANA)}

YANA features a platform-independent, dedicated toolbox for metabolic networks with a graphical user interface to calculate (integrating METATOOL), edit (including support for the SBML format), visualize, centralize, and compare elementary flux modes. Further, YANA calculates expected flux distributions for a given Elementary Mode (EM) activity pattern and vice versa. As data are noisy, YANA features a fast evolutionary algorithm (EA) for the prediction of EM activities with minimum error, including alerts for inconsistent experimental data (Schwarz et al., 2005).

EMA is an algorithm that systematically enumerates all possibilities how enzymes can operate together without violating the steady state condition of the system. Using EMA, complex networks can be analyzed in terms of contained pathways, robustness, central enzymes, medical targets, optimum yield and effector compounds, such as signaling phospholipids, with interesting applications in medicine and biotechnology (Gerst et al., 2015). YANA offers and implements strategies to reduce network complexity. YANA also involves a dissection algorithm, a centralization algorithm and an average diameter routine which are used to simplify and analyze complex networks.

$$
\begin{aligned}
& F\left(E_{j}\right)=\sum_{i=1}^{N}\left|f_{i}^{j}\right| \times a_{i} \\
& F\left(E_{j}\right)=\sum_{i=1}^{N} f_{i}^{j} \times a_{i}
\end{aligned}
$$

YANA offers the possibility to compute flux distributions both in absolute (ignoring reaction directions) and relative values. This is done by iterating over all calculated modes, and summing the absolute (eq.1) or relative (eq. 2) flux coefficients of each enzyme multiplied with the activity of the EM. 


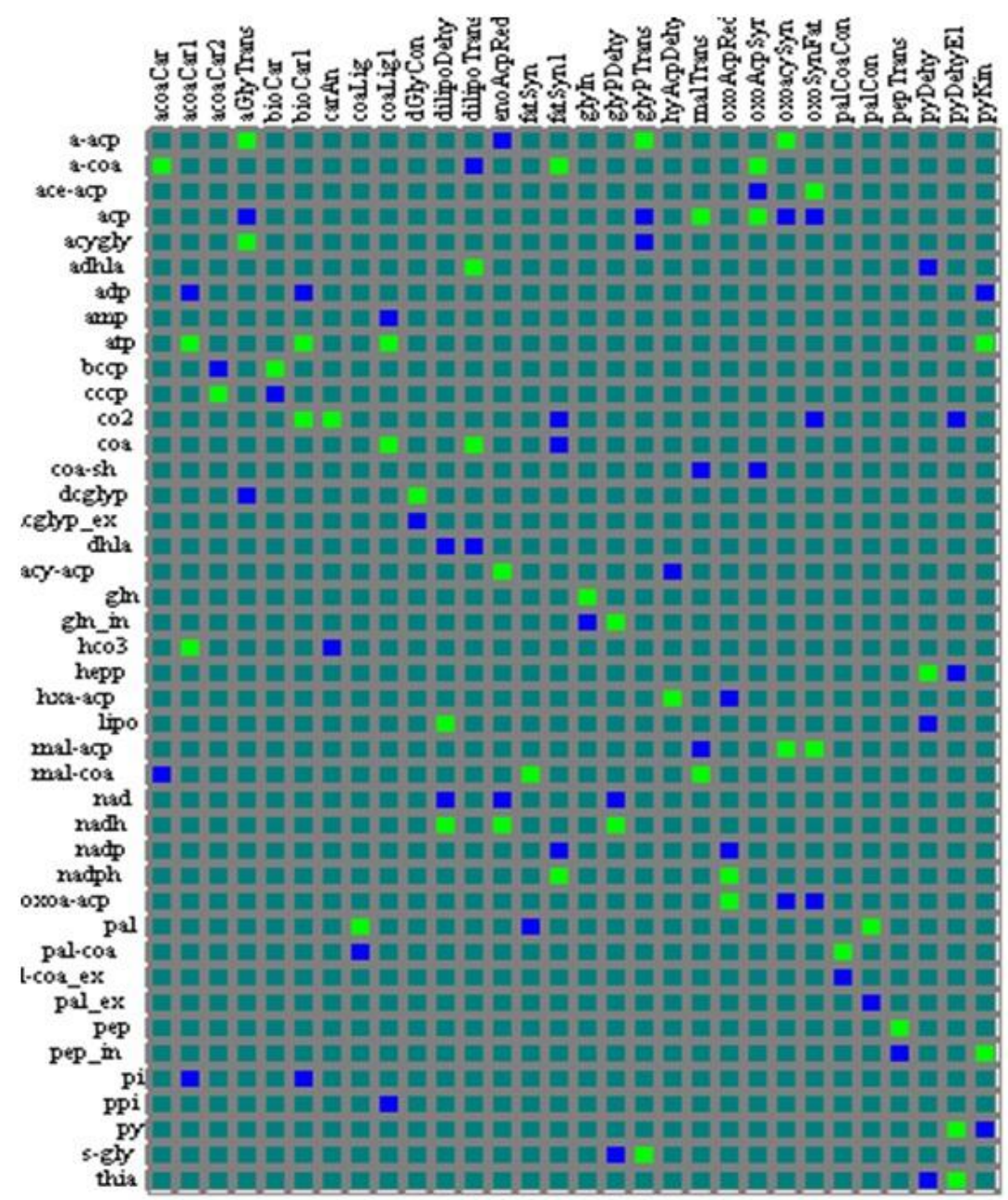

Fig. 1: Stoichiometric matrix of Fatty acid pathway.

\section{RESULTS AND DISCUSSION}

Apart from using the host lipids source, parasites like plasmodium are competent enough to synthesize lipid de-novo from simple metabolites. One of the important rate limiting steps in the pathway is the Acetyl-CoA Carboxylase reaction which catalyses the first committed step of the pathway, i.e. formation of Malonyl-CoA. However, it cannot be preferred as a malarial target due to its genome homology with humans. The apicoplast FAS II is dramatically different from the animal FAS I, both in overall structure and in the sequence of individual functional domains, greatly increasing the chances of successful development of specific inhibitors. These FAS II enzymes are taxonomically related to algal enzymes so they can be preferred targets (Gornicki 2003). The co-efficient of fatty acid pathway reactions was shown in a simplified representation through stoichiometric matrix in Fig.1. Here, the metabolites are displayed as row and enzymes as columns in the matrix.

\section{Elementary mode analysis}

Elementary modes are minimal set of enzymes that could operate at steady state with all irreversible reactions proceeding in the appropriate direction. Robustness of the pathway is its capability to function in presence of external and internal perturbations.

These tools can be used to carry out enzyme knockout analysis and compare the flux distribution values of each knockout with the normal case. This information can be used to find the effect of each enzyme on the pathway and thus recognize a possible drug target. Calculating flux distributions from a given theoretical set of EM activities is important e.g. to estimate the relevance of an enzyme in a metabolic network, but it would be a desirable goal if one could somehow measure flux distributions in living cells and map them onto the EM activities . It would then be possible to take an experimental snapshot of the system and from it derive actual pathway activities (Schwarz et al., 2005). EMA is a useful metabolic pathway analysis tool to identify the structure of a metabolic network that links the cellular phenotype to the corresponding genotype. EMA helps in analyzing metabolic network properties like structure, robustness, fragility and regulation of the network. The activity, Flux sum, path length along with the individual EFM was shown in numbers with $100 \%$ activity in Fig 2. 


\begin{tabular}{|c|c|c|c|c|c|}
\hline 11 & Activity & Flux sum & Reversible? & Pathlength & Reactions \\
\hline & $100 \%$ & 2 & $v$ & & 2 (1 aGlyTrans) (-1 glyPTrans) \\
\hline & $100 \%$ & 1 & $v$ & & 1 (1 hyAcpDehydra) \\
\hline & $100 \%$ & 2 & $\square$ & & 2 (-1 dilipoDehy) (1 glyPDehy) \\
\hline & $100 \%$ & 1 & $\square$ & & 1 (1 biocan) \\
\hline & $100 \%$ & 2 & $\square$ & & 2 (-1 coaLig) (1 palCon) \\
\hline & $100 \%$ & 1 & $\square$ & & 1 (1 pyDehy) \\
\hline & $100 \%$ & 1 & $\square$ & & 1 (1 palcoacon) \\
\hline & $100 \%$ & 3 & $\square$ & & 3 (1 carAn) (1 dilipoTrans) (1 fatSyn1) \\
\hline & $100 \%$ & 1 & $\square$ & & 1 (1 gly/n) \\
\hline 10 & $100 \%$ & 1 & $\square$ & & 1 (1 dGlyCon) \\
\hline 11 & $100 \%$ & 1 & $\square$ & & 1 (1 acoaCar2) \\
\hline 12 & $100 \%$ & 7 & $\square$ & & 7 (1 acoaCar) (-1 aGlyTrans) (1 coaLig) (1 dilipoDehy) (-1 enoAcpRed) (1 fatSyn) (-1 oxoAcps \\
\hline 131 & $100 \%$ & 7 & $\square$ & & 7 (1 acoaCar) (1 coaLig) (1 dilipoDehy) (-1 enoAcpRed) (1 fatSyn) (-1 glyPTrans) (-1 oxoAcps \\
\hline 14 & $100 \%$ & 7 & $\square$ & & 7 (1 acoaCar) (-1 aGlyTrans) (1 coaLig) (-1 enoAcpRed) (1 fatSyn) (1 glyPDehy) (-1 oxoAcpS) \\
\hline 151 & $100 \%$ & 7 & $\square$ & & 7 (1 acoaCar) (1 coaLig) (-1 enoAcpRed) (1 fatSyn) (1 glyPDehy) (-1 glyPTrans) (-1 oxoAcpS) \\
\hline 161 & $100 \%$ & 7 & $\square$ & & 7 (1 acoaCar) (-1 aGlyTrans) (1 dilipoDehy) (-1 enoAcpRed) (1 fatSyn) (-1 oxoAcpSyn) (1 pal \\
\hline 17 & $100 \%$ & 7 & $\square$ & & 7 (1 acoaCar) (1 dilipoDehy) (-1 enoAcpRed) (1 fatSyn) (-1 glyPTrans) (-1 oxoAcpSyn) (1 palk \\
\hline 181 & $100 \%$ & 7 & $\square$ & & 7 (1 acoaCar) (-1 aGlyTrans) (-1 enoAcpRed) (1 fatSyn) (1 glyPDehy) (-1 oxoAcpSyn) (1 palC \\
\hline 19 & $100 \%$ & 7 & $\square$ & & 7 (1 acoaCar) (-1 enoAcpRed) (1 fatSyn) (1 glyPDehy) (-1 glyPTrans) (-1 oxoAcpSyn) (1 palci, \\
\hline 201 & $100 \%$ & 3 & $\square$ & & 3 (-1 dilipoTrans) (-1 fatSyn1) (1 pyDehyE1) \\
\hline 21 & $100 \%$ & 2 & $\square$ & & 2 (1 carAn) (1 pyDehyE1) \\
\hline 22 & $100 \%$ & 1 & $\square$ & & 1 (1 pepTrans) \\
\hline 23 & $100 \%$ & 6 & $\square$ & & 6 (-1 dilipoDehy) (1 dilipoTrans) (1 enoAcpRed) (1 fatSyn1) (1 oxoacySyn) (-1 oxoSynfatSyn) \\
\hline 24 & $100 \%$ & 9 & $\square$ & & 9 (1 acoaCar) (-1 aGlyTrans) (1 coaLig) (1 dilipoTrans) (1 fatSyn) (1 fatSyn1) (-1 oxoAcpSyn) \\
\hline 25 & $100 \%$ & 9 & $\square$ & & 9 (1 acoaCar) (1 coaLig) (1 dilipoTrans) (1 fatSyn) (1 fatSyn1) (-1 glyPTrans) (-1 oxoAcpSyn) \\
\hline & $100 \%$ & 9 & $\square$ & & 9 (1 acoaCar) (-1 aGlyTrans) (1 dilipoTrans) (1 fatSyn) (1 fatSyn1) (-1 oxaAcpSyn) (1 oxoacyS \\
\hline
\end{tabular}

Fig. 2: EFMs of the fatty acid biosynthesis with $100 \%$ efficiency.

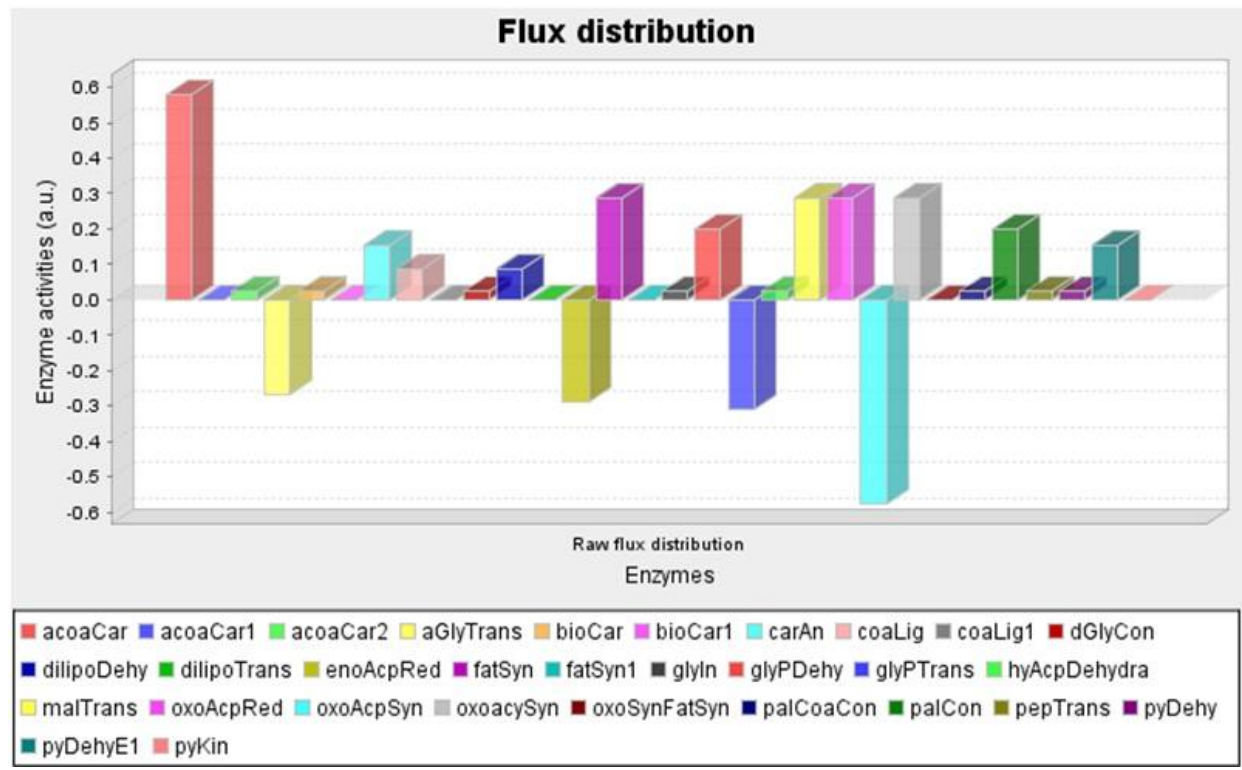

Fig. 3: Flux distribution with enzyme activities.

\section{EFM Normal Case}

The EFMs was obtained by using Metatool with YANA square as front end. Fig. 3 indicates the normal case enzymes behavior.

The graphical representation of EFM activity number 23 with a path length of 6 was shown in Fig.4 depict the various reactions involved. These reactions are the essential ones which determine the functioning of the pathway. The distance graph also shows how close the metabolites of a given EFM are to each other. The yellow boxes depict the reaction nodes and are denoted by the enzyme names. The balls depict the connection nodes and are denoted by metabolites. The red balls are external metabolites and the blue ones are internal metabolites. The length of the shortest path between two reactions is considered as the measure of the functional distance. The EMFs shown here contain metabolites and enzymes which are used further in knockout analysis.

\section{CASE-1: Key Enzyme Knock out (individual)}

After studying the various EFMs, we concluded some of the essential enzymes of the pathway. These enzymes were then knocked out one by one and the resulting flux distribution graph and values were noted. These values gave a fair idea of the effect of each enzyme on the overall functioning of the pathway. 


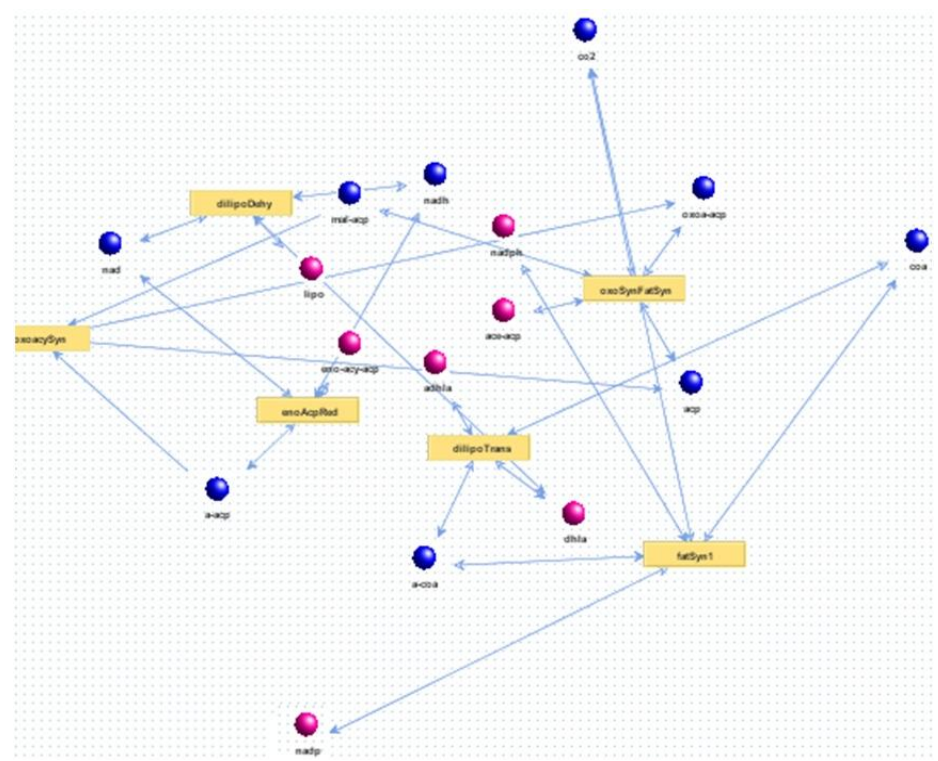

Fig.4: Path length graph for EFM number 23.

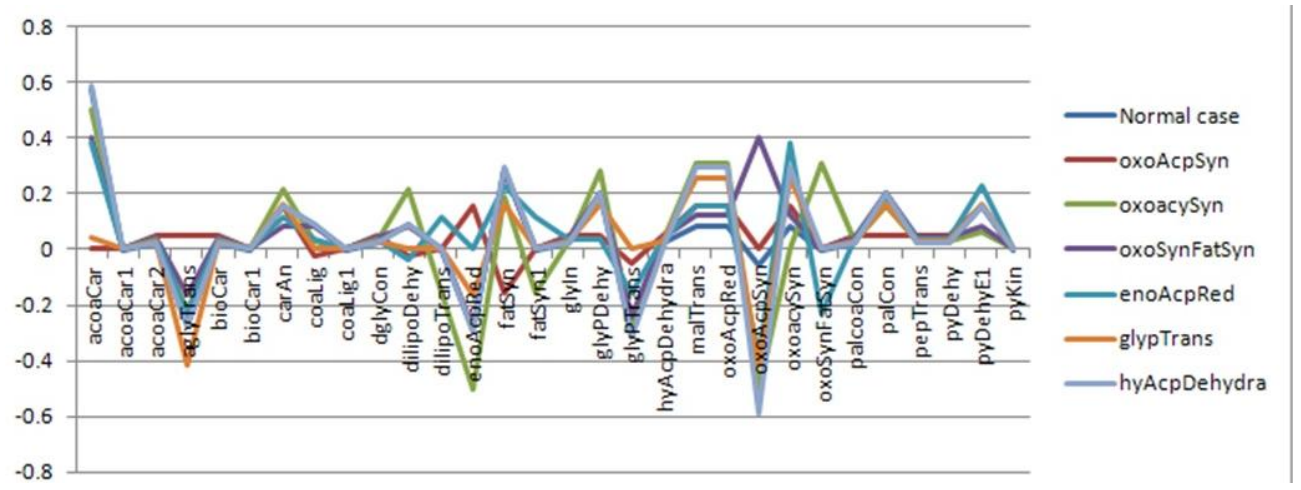

Fig. 5: Flux values for Normal case vs. individual enzyme knockout cases.

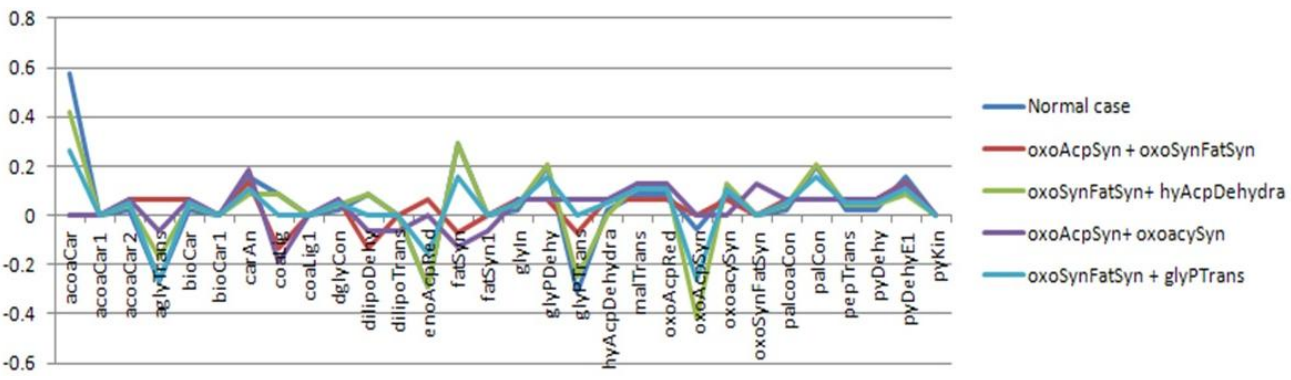

Fig. 6: Flux values for Normal case vs. double enzyme knockout cases.

The enzymes studied were 3-oxoacyl-ACP synthase (oxoacpSyn), 3-oxoacyl-ACP synthase fatty acid synthase (oxoSynFatSyn, oxoacySyn), enoyl-ACP reductase (enoAcpRed), glycerol-3phosphate o-acyl transferase (glypTrans) and 3-hydroxyacylACP dehydratase (hyAcpDehydra). The flux distribution chart for the $100 \%$ activity (Normal case) of the network is compared with the flux distribution values after each enzyme knockout case (Fig. 5).

\section{CASE- 2: Multiple Enzyme Knockout case}

In this scenario, we knocked out a combination of two enzymes at the same time. This resulted in further variation of the flux distribution values, showing that multi-enzyme knock out gave relatively better results than single enzyme knock out cases with overall reduction in flux flow (Fig.6).

\section{Fatty Acid Pathway Robustness Report}

The robustness of the fatty acid biosynthesis pathway was obtained from YANA square in the Analysis Mode. This value shows good capability of the pathway to adjust to external and internal perturbations. The robustness was studied for the various knockout cases. This value was used to conclude the most effective knockout case for controlling this key pathway. Along 
with the robustness, we also obtain average modes loss and average production loss values as shown in Table.1.

Table 1: Robustness percentage in different knockout cases.

\begin{tabular}{llll}
\hline \multicolumn{1}{c}{ Knockout cases } & $\begin{array}{c}\text { Avg } \\
\text { production } \\
\text { loss }\end{array}$ & $\begin{array}{c}\text { Robustn } \\
\text { ess }\end{array}$ & $\begin{array}{c}\text { Avg } \\
\text { modes } \\
\text { lost }\end{array}$ \\
\hline glyPTrans & 0.41 out of 13 & $96.82 \%$ & 5.41 \\
oxoAcpSyn & 0.28 out of 12 & $97.7 \%$ & 1.83 \\
oxoacySyn & 0.41 out of 12 & $98.85 \%$ & 5.76 \\
oxoSynFatSyn & 0.79 out of 13 & $93.9 \%$ & 3.34 \\
enoAcpRed & 0.1 out of 13 & $99.2 \%$ & 3.34 \\
hyAcpDehydra & 0.66 out of 13 & $94.96 \%$ & 9.1 \\
oxoAcpSyn + oxoSynFatSyn & 0.46 out of 11 & $95.8 \%$ & 1.04 \\
oxoSynFatSyn + hyAcpDehydra & 0.82 out of 13 & $93.68 \%$ & 3.43 \\
oxoAcpSyn + oxoacySyn & 0.86 out of 13 & $93.41 \%$ & 1.25 \\
oxoSynFatSyn +glyPTrans & 0.86 out of 13 & $93.41 \%$ & 2.18 \\
\hline
\end{tabular}

In case of $100 \%$ enzyme activity, the robustness of the pathway is $95.38 \%$. During the knock- out of oxoSynFatSyn enzyme, the robustness reduces to $93.9 \%$ whereas during the knockout of enoAcpRed it increases to $99.2 \%$. In case of multiple enzyme knockouts, the robustness shows further reduction sliding down to $93.41 \%$. This has occurred in case of oxoAcpSyn with oxoacySyn and oxoSynFatSyn with glyPTrans double knock-out. This shows that the pathway is very robust in general. However, targeting multiple enzymes would reduce the overall flux and robustness resulting in more effectiveness in controlling this pathway.

\section{CONCLUSION}

The topological analysis of fatty acid biosynthesis occurring in apicoplast of Plasmodium falciparum was done using the elementary flux modes which act as a parameter for enzyme knockout cases. This analysis also uses flux distribution values and the comparison between the normal and the knocked out cases. Robustness of the pathway is the main parameter considered here for finding the effect of the knocked out enzymes on the biochemical pathway. These enzymes can be potential drug targets and can be used to treat cerebral malaria. We have concluded that knocking out multiple enzymes at the same time has more effect on reducing the robustness of the pathway. From this we conclude that, knocking out oxoAcpSyn(3-oxoacyl-ACP synthase) with oxoacySyn(3-oxoacyl-ACP synthase) and oxoSynFatSyn(3oxoacyl-ACP synthase) with glyPTrans(Glycerol-3-phosphate oacyl transferase) results in the pathway showing minimal robustness. Out of the above mentioned four enzymes, the first three are established drug targets. Nonetheless, their effect on stopping the fatty acid synthesis would be better when they are combined together than when they are knocked out (inhibited) alone. Thus, we propose multi drug targeting of these identified targets as a better way to treat brain malaria.

\section{REFERENCES}

Aly ASI, Vaughan AM, Kappe SHI. Malaria Parasite Development in the Mosquito and Infection of the Mammalian Host. Annual review of microbiology, 2009;63:195-221.

Ben Mamoun C, Prigge ST, Vial H. Targeting the Lipid Metabolic Pathways for the Treatment of Malaria. Drug development research, 2010;71(1):44-55.

Das A, Sharma M, Gupta B, Dash AP. Plasmodium falciparum and Plasmodium vivax: so similar, yet very different. Parasitol Res, 2009 , 105(4):1169-71.

Gerst MP, Ruckerbauer DE, Mattanovich D, Jungreuthmayer C, Zanghellini J.Metabolomics integrated elementary flux mode analysis in large metabolic networks. Sci Rep, 2015; 10;5:8930.

Gornicki P.Apicoplast fatty acid biosynthesis as a target for medical intervention in apicomplexan parasites. Int J Parasitol, 2003; 33:885-96.

Kauffman KJ, Prakash P, Edwards JS. Advances in flux balance analysis.Curr Opin Biotechnol, 2003; 14(5):491-6.

Lim L, McFadden GI. The evolution, metabolism and functions of the apicoplast. Philosophical Transactions of the Royal Society B: Biological Sciences, 2010;365(1541):749-763.

Pfeiffer T, Sánchez-Valdenebro I, Nuño JC, Montero F, Schuster S.METATOOL: for studying metabolic networks. Bioinformatics, 1999; 15(3): 251-7.

Papin JA, Price ND, Wiback SJ, Fell DA, Palsson BO.Metabolic pathways in the post-genome era. Trends Biochem Sci, 2003; 28(5):250-8.

Qidwai T1, Khan F. Antimalarial drugs and drug targets specific to fatty acid metabolic pathway of Plasmodium falciparum. Chem Biol Drug Des, 2012; 80(2):155-72.

Schwarz R, Musch P, von Kamp A, Engels B, Schirmer H, Schuster S, Dandekar T.YANA - a software tool for analyzing flux modes, gene-expression and enzyme activities. BMC Bioinformatics, 2005; 1(6): 135 .

Waller RF, Ralph SA, Reed MB, et al. A Type II Pathway for Fatty Acid Biosynthesis Presents Drug Targets in Plasmodium falciparum. Antimicrobial Agents and Chemotherapy, 2003; 47(1):297-301.

\section{How to cite this article:}

Meenakshi Pradhan, Arnold Emerson I, Kiruba Thangam R, Febin Prabhu Dass J. Exploring Novel Drug Targets in Fatty Acid Pathway of Plasmodium Falciparum. J App Pharm Sci, 2015; 5 (10): 107-112. 